\title{
Delay-Based Regularization for ECG Imaging of Transmembrane Voltages
}

\author{
Steffen Schuler ${ }^{1}$, Danila Potyagaylo ${ }^{2}$, Olaf Dössel ${ }^{1}$ \\ ${ }^{1}$ Institute of Biomedical Engineering, Karlsruhe Institute of Technology (KIT), Karlsruhe, Germany \\ ${ }^{2}$ EP Solutions SA, 1400 Yverdon-les-Bains, Switzerland
}

\begin{abstract}
We suggest a new regularization method for reconstruction of cardiac transmembrane voltages (TMV) from body surface potentials that is based on imposing similarity between time-aligned TMVs. An iterative scheme is proposed to update the delays needed for time-alignment. Evaluation of the method using simulated ventricular pacings showed a clear improvement over second order Tikhonov.
\end{abstract}

\section{Introduction}

Electrocardiographic imaging combines a volume conductor model of the torso with measurements of body surface potentials (BSP) to image the electrical activity of the heart. This technique may become a powerful tool for non-invasive detection of electrophysiological properties related to arrhythmias. As potentials caused by cardiac sources are strongly attenuated and blurred by the torso, the inverse problem of reconstructing these sources from BSPs is ill-posed and requires regularization. While the propagation of potentials from the heart to the torso is quasi-static, the spread of electrical excitation across the heart is a highly dynamic and spatio-temporally coherent process. Therefore, including time in formulations for solving the inverse problem has long been seen as a way to facilitate the uniqueness of reconstructed signals. In [1], for example, signals were constrained to simultaneously be smooth in space and time. Others have enforced physiologically motivated constraints on the temporal evolution of cardiac sources [2]. However, only few approaches have tried to directly link space and time in a way that is specific to the spread of cardiac excitation [3].

In this work, we propose a novel, iterative regularization scheme that establishes such a spatio-temporal link by imposing similarity between time-aligned transmembrane voltages (TMV) of nearby nodes. The basic idea behind this method is the same as for the "wave-equation based" interpolation in [4]. There, cardiac potentials are first shifted in time by their negative activation times and spatial interpolation is then applied to these time-aligned potentials. Both methods make use of the observation that the morphology of cardiac signals usually varies little within a local neighborhood and signals differ mainly by a time shift. Given the stereotypical time course of an action potential, this should especially be true for TMVs.

\section{Methods}

\subsection{Delay-based regularization}

The delay-based regularization is given by:

$$
\begin{aligned}
\overline{\mathbf{x}} & =\arg \min _{\overline{\mathbf{x}}}\left(\|\overline{\mathbf{A}} \overline{\mathbf{x}}-\overline{\mathbf{b}}\|_{2}^{2}+\lambda_{L}\|\overline{\mathbf{L}} \overline{\mathbf{x}}\|_{2}^{2}+\lambda_{D}\|\mathbf{D} \overline{\mathbf{x}}\|_{2}^{2}\right) \\
& \Leftrightarrow \quad\left(\overline{\mathbf{A}}^{\top} \overline{\mathbf{A}}+\lambda_{L} \overline{\mathbf{L}}^{\top} \overline{\mathbf{L}}+\lambda_{D} \mathbf{D}^{\top} \mathbf{D}\right) \overline{\mathbf{x}}=\overline{\mathbf{A}}^{\top} \overline{\mathbf{b}}
\end{aligned}
$$

We use the notation from [1] to include time in the formulation. Overlines indicate block diagonal matrices or block vectors:

$$
\begin{gathered}
\overline{\mathbf{A}}=\mathbf{I}_{T} \otimes \mathbf{A}, \quad \overline{\mathbf{L}}=\mathbf{I}_{T} \otimes \mathbf{L} \\
\overline{\mathbf{x}}=\left[\mathbf{x}_{1}^{\top}, \ldots, \mathbf{x}_{T}^{\top}\right]^{\top}, \quad \overline{\mathbf{b}}=\left[\mathbf{b}_{1}^{\top}, \ldots, \mathbf{b}_{T}^{\top}\right]^{\top}
\end{gathered}
$$

$T$ is the number of timesteps, $\mathbf{I}_{T}$ is a $T \times T$ identity matrix and $\otimes$ denotes the Kronecker product. $\mathbf{A}$ is the lead field matrix transforming TMVs $\mathbf{x}$ into BSPs $\mathbf{b}$ and $\mathbf{L}$ is an approximation of the Laplacian operator.

The last summand in (1) respresents the new delay-based constraint. $\mathbf{D}$ is a $P \cdot T \times T \cdot N$ matrix, where $N$ is the number of nodes and $P$ is the number of pairs of nearby nodes. For every node pair, this matrix calculates the differences of signals after alignment in time using previously determined delays. These delays between nodes of each pair are updated iteratively as will be described in section 2.1.3. $\mathrm{D}$ has to be reassembled for every new set of delays.

The MATLAB code used in this work is available in [5].

\subsubsection{Theoretical considerations}

According to bidomain theory [6], the gradient of TMVs is the source of BSPs. Spatially constant offsets of TMVs are therefore in the null-space of $\mathbf{A}$ and cannot be recovered with the static inverse problem, i.e. by reconstructing each timestep independently. A consequence is that the time course of reconstructed TMVs is not (directly) meaningful, as each timestep might be superimposed by a different, arbitrary offset. A unique result of the timeindependent problem can only be obtained by directly penalizing the amplitude of TMVs, for example using a zero order Tikhonov constraint. However, this results in unphysiological time courses. For example, the spatial gradient of TMVs is small during plateau phase and thus such a constraint would force the TMV to zero during this period. 
The delay-based constraint however, links time and space in a way that allows to recover such null-space components. Suppose, for example, the spatial gradient of TMVs is zero. For each timestep, any spatially constant value of TMVs would thus be allowed by the term $\|\overline{\mathbf{A}} \overline{\mathbf{x}}-\overline{\mathbf{b}}\|_{2}^{2}$. However, such an arbitrary, time-changing value would lead to deviations of time-shifted signals and is thus not permitted, as it leads to a large value of $\|\mathbf{D} \overline{\mathbf{x}}\|_{2}^{2}$. In other words, the temporal change of spatially constant offsets is made unique by the delay-based constraint. What remains undetermined is a spatially and temporally constant offset. Following the same line of reasoning, a common deflection in time-aligned TMVs will lead to a spatial gradient in nonaligned TMVs that has to fit to the given BSPs. From theoretical considerations, the delay-based constraint therefore adds a regularization that is particularly effective for TMVs, while supporting their physiological properties.

\subsubsection{Solving the linear system}

Equation (1) is a system of $T \cdot N$ linear equations and can become very large, if many timesteps or many nodes are used. However, since the left-hand-side matrix is sparse, symmetric and positive definite, it can be solved efficiently using preconditioned conjugate gradient (PCG). The following approximate inverse was found to work well as a preconditioner $\left(\mathbf{I}_{N}\right.$ is an $N \times N$ identity matrix):

$$
\overline{\mathbf{M}}=\mathbf{I}_{T} \otimes \mathbf{M}, \quad \mathbf{M}=\left(\mathbf{A}^{\top} \mathbf{A}+\lambda_{L} \mathbf{L}^{\top} \mathbf{L}+\lambda_{D} \mathbf{I}_{N}\right)^{-1}
$$

We use the MATLAB implementation of PCG with function handles replacing both the coefficient matrix and the preconditioner matrix. This allows to save a lot of memory, as the block diagonal matrices do not have to be created explicitly. Instead, $\overline{\mathbf{x}}$ is reshaped into a matrix $\mathbf{X}$ and multiplications with $\left(\mathbf{A}^{\top} \mathbf{A}+\lambda_{L} \mathbf{L}^{\top} \mathbf{L}\right)$ in (1) and $\mathbf{M}$ in (2) are performed as a matrix product (note the missing overlines). Only the multiplication with $\mathbf{D}^{\top} \mathbf{D}$ in (1) has to be done using the block vector form $\overline{\mathbf{x}}$. As $\mathbf{D}$ has only up to $2 \cdot P \cdot T$ nonzero elements, this is not a problem.

\subsubsection{Iterative update of delays and choice of regularization parameters}

Our aim is to get a solution that is predominantly regularized by the delay-based constraint. However, optimal delays are not known beforehand. Therefore, we perform an initial reconstruction using second order Tikhonov, which is equivalent to setting $\lambda_{D}$ to zero in (1). Delays are determined from this initial solution as described in the next section and updated iteratively from solutions obtained by gradually letting $\lambda_{L}$ go to zero and increasing $\lambda_{D}$. The initial parameter $\lambda_{L, 0}$ is found using the L-curve method by solving this equation for multiple values of $\lambda_{L}$ :

$$
\mathbf{X}=\arg \min _{\mathbf{X}}\left(\|\mathbf{A X}-\mathbf{B}\|_{F}^{2}+\lambda_{L}\|\mathbf{L} \mathbf{X}\|_{F}^{2}\right)
$$

Here, $\mathbf{X}$ and $\mathbf{B}$ contain nodes along columns and timesteps along rows. To avoid oversmoothing, we only include the time span of depolarization to determine the L-curve.

Throughout iterations, $\lambda_{L}$ is reduced according to a smooth function and $\lambda_{D}$ is increased. Solving (1) many times with a fixed $\lambda_{L}$ to obtain an L-curve for $\lambda_{D}$ is computationally demanding. Therefore, we use a different approach: $\lambda_{D}$ is adjusted, so that the data misfit term $\|\overline{\mathbf{A}} \overline{\mathbf{x}}-\overline{\mathbf{b}}\|_{2}$ is held constant. This is achieved by finding the root of the following residual using the secant method:

$r_{i}\left(\lambda_{D}^{\prime}\right)=\left\|\overline{\mathbf{A}} \overline{\mathbf{x}}_{i}\left(\lambda_{D}^{\prime}\right)-\overline{\mathbf{b}}\right\|_{2}-\left\|\overline{\mathbf{A}} \overline{\mathbf{x}}_{0}-\overline{\mathbf{b}}\right\|_{2}, \quad \lambda_{D}^{\prime}=\log \lambda_{D}$

$\overline{\mathbf{x}}_{0}$ is the initial solution obtained for $\lambda_{L}=\lambda_{L, 0}, \lambda_{D}=0$. To further speed up computations, $\lambda_{D}^{\prime}$ is extrapolated quadratically from previous iterations before running the secant method. Moreover, the previous solution $\overline{\mathbf{x}}_{i-1}$ is used as initial estimate for PCG.

Experiments showed that it is beneficial to let $\lambda_{L}$ slowly approach zero with a slope of zero. Therefore, we chose the following function to reduce $\lambda_{L}$ across 100 iterations:

$$
\lambda_{L, i}=(1-i / 99)^{10} \lambda_{L, 0}, \quad i=0, \ldots, 99
$$

\subsubsection{Estimation of delays}

Delays are estimated by applying the global activation times approach from [7] to reconstructed TMVs. This method uses a least-squares regression to obtain activation times (AT) that best fit to many inter-node delays determined using cross-correlation. We first apply a temporal Gaussian filter (order 2, $\sigma=12 \mathrm{~ms}$ ) to TMVs. As signal for cross-correlation, we then use the product of the surface gradient norm and the temporal derivative, as originally suggested for deflection-based AT estimation in [8]. Delays for new reconstructions are then calculated as differences of corresponding ATs.

As described in section 2.1.1, an arbitrary, spatially constant offset can be present in TMVs when reconstructed with $\lambda_{D}=0$. Before AT estimation, we thus perform a baseline correction based on spatial statistics: The central timesteps of de- and repolarization $t_{d}$ and $t_{r}$ are found using the median absolute deviation (MAD). Depending on whether the lower or upper bound of TMVs should be better defined during a time interval, the baseline $g(t)$ is then estimated using the lower or upper percentile (lp: $10^{\text {th }}$, up: $90^{\text {th }}$ percentile). $g(t)$ is finally subtracted from TMVs.

$$
\begin{aligned}
t_{d} & =\text { time of largest peak in } \operatorname{MAD}(\mathbf{x}(t)) \\
t_{r} & =\text { time of second largest peak in } \operatorname{MAD}(\mathbf{x}(t)) \\
g(t) & =\left\{\begin{array}{l}
\operatorname{lp}(\mathbf{x}(t)) \\
\operatorname{up}(\mathbf{x}(t))-\operatorname{up}\left(\mathbf{x}\left(t_{d}\right)\right)+\operatorname{lp}\left(\mathbf{x}\left(t_{d}\right)\right), t_{d} \leq t<t_{r} \\
\operatorname{lp}(\mathbf{x}(t))-\operatorname{lp}\left(\mathbf{x}\left(t_{r}\right)\right)+\operatorname{up}\left(\mathbf{x}\left(t_{r}\right)\right) \ldots \\
-\operatorname{up}\left(\mathbf{x}\left(t_{d}\right)\right)+\operatorname{lp}\left(\mathbf{x}\left(t_{d}\right)\right), t \geq t_{r}
\end{array}\right.
\end{aligned}
$$




\subsection{Evaluation using simulations}

To evaluate the delay-based regularization, we applied it for reconstruction of three simulated ventricular pacings at the LV and RV lateral wall and the RV septum. Anisotropic excitation simulations were performed using bidomain and ten Tusscher models. Endo- and epicardial fiber angles were set to $\pm 60^{\circ}$ and cell model variants for endo, mid and epi layers were used. The boundary element method and a homogeneous torso model with 200 electrodes (Fig. 1) were used for forward calculations. The same forward model was used for inverse calculations, but white Gaussian noise was added to BSPs (mean signal-to-noise ratio: $10 \mathrm{~dB}$ ). The ventricular surface mesh consisted of 2306 nodes (mean edge length: $6.2 \mathrm{~mm}$ ). Node pairs for the delay-based constraint and for AT estimation were defined as all pairs with a shortest path distance of 2 edges. The transmural extension from [9] was used for the Laplacian. As evaluation metrics, we use the Pearson correlation coefficient (CC) and the mean absolute error (MAE) for ATs, and the spatial and temporal CC (SCC, TCC) for TMVs.
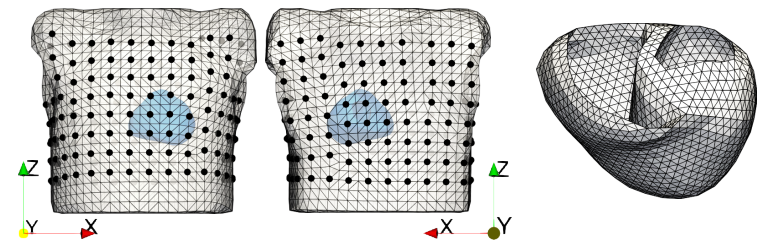

Figure 1. Geometries for forward and inverse calculations.

\section{Results}

Fig. 2 shows the four metrics plotted over iterations. For all three pacings, ATs gradually improve until iteration 80 , where CCs and MAEs reach a plateau. A similar improvement over iterations can be seen for TMV correlations.
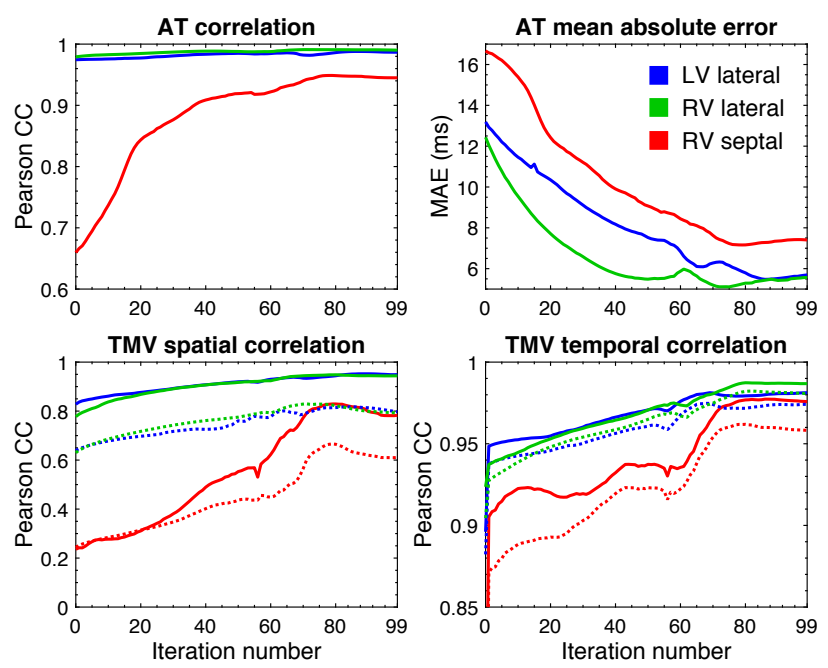

Figure 2. Metrics over iterations. Bottom: Solid lines: median; dotted lines: mean.
For lateral pacings, the AT pattern obtained with second order Tikhonov (iteration 0 ) already matches well with the truth (Fig. 3). However, more details are recovered with the delay-based regularization (iteration 99) and the MAE is more then halved. For the septal pacing, differences are more pronounced (Fig. 4). At iteration 0, the site of earliest activation is wrongly indicated at the RV anterior wall and ATs on the posterior side show large deviations from the truth. The AT map of iteration 99 is much more precise and the excitation origin is correctly indicated at the RV septum. This is also reflected in the metrics: The AT $\mathrm{CC}$ is increased from 0.659 to 0.945 .

TMV patterns for the septal pacing are depicted in Fig. 5. At iteration 0 , the wavefront is strongly blurred and shifted. Throughout iterations, it moves to the correct location and gets sharper. Eventually, it is centered around its true origin and the epicardial breakthrough occurs at the right spot.

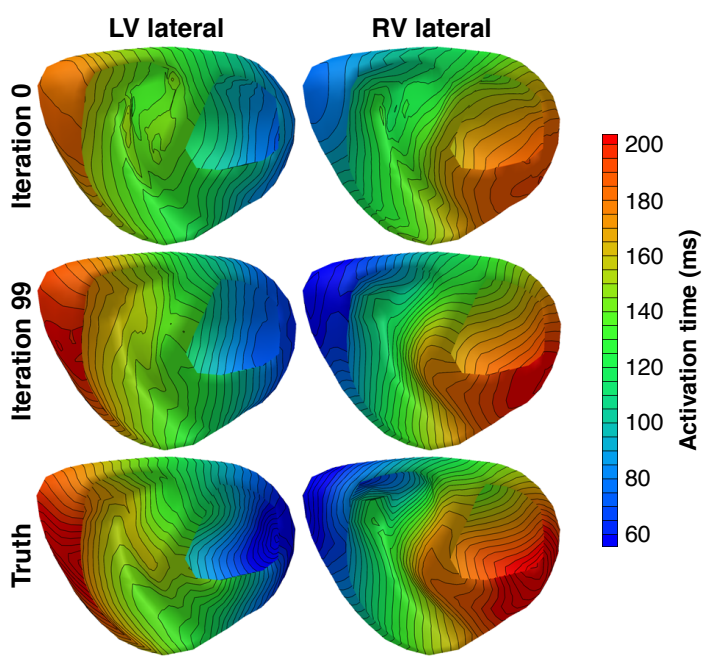

Figure 3. ATs for lateral pacings.

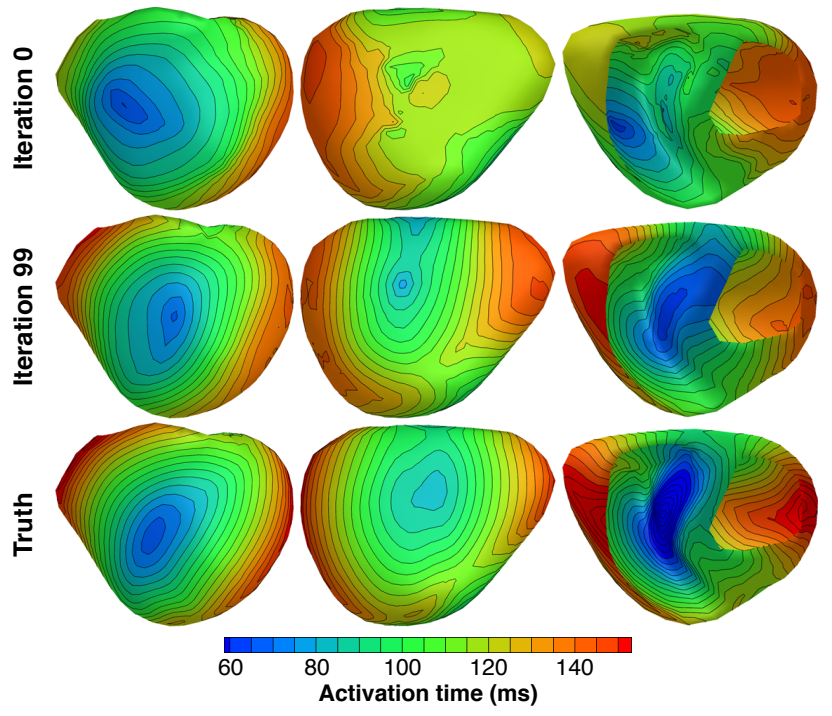

Figure 4. ATs for the RV septal pacing. 
Fig. 6 shows exemplary TMV time courses for RV cases. Although initial TMVs were baseline-corrected, their time courses often show wrong deflections. Across iterations, these deflections are attenuated and only one clear deflection remains for de- and repolarization. Noise is also much better suppressed by the delay-based regularization.

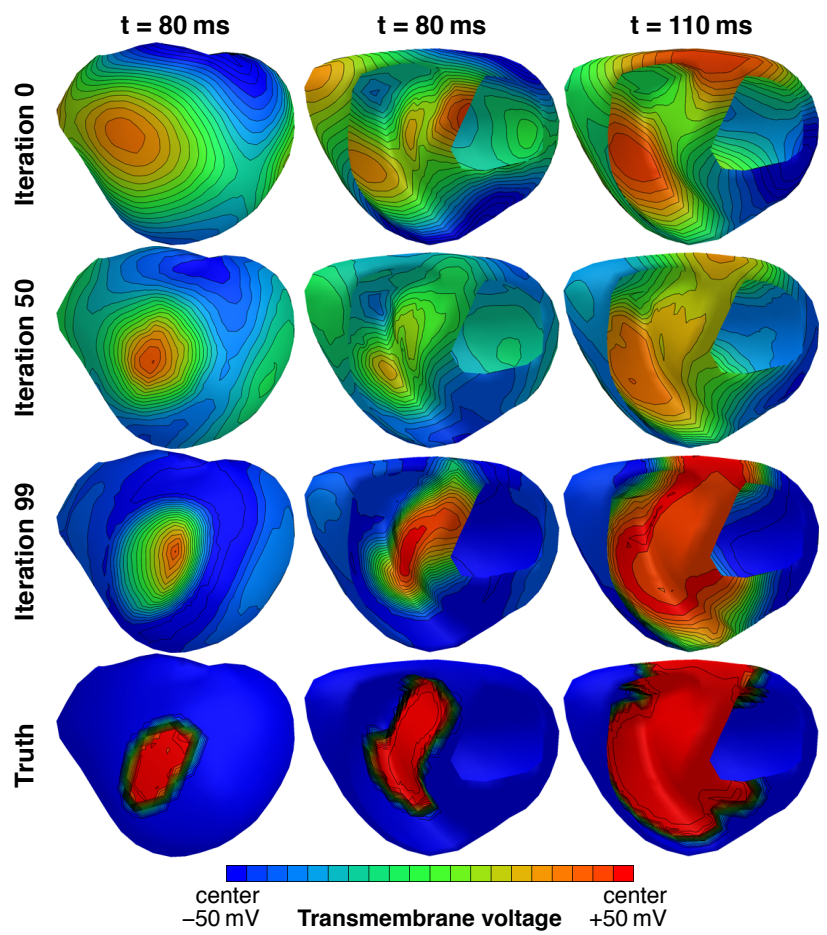

Figure 5. TMVs for two timesteps of the septal pacing.

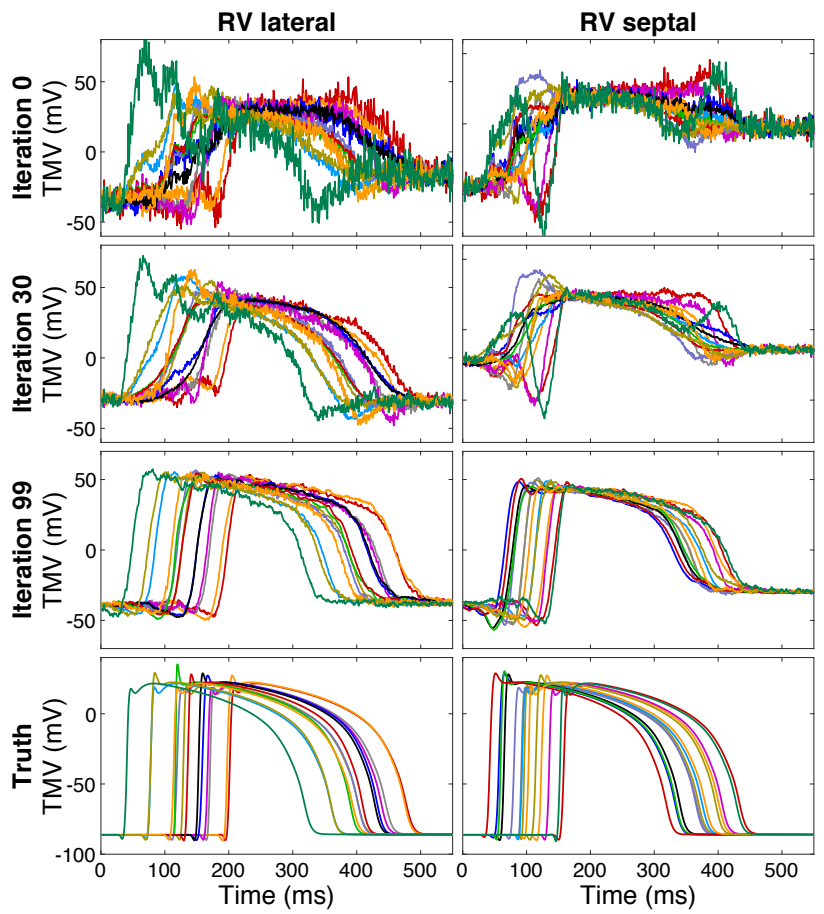

Figure 6. Exemplary TMV time courses for RV pacings.

\section{Discussion and conclusion}

Simulations showed that delay-based regularization substantially improves reconstructions with respect to all considered metrics when compared to second order Tikhonov. Improvement was largest for the most problematic case of an RV septal pacing. While simulations accounted for anisotropy and transmural APD heterogeneity, they did not include scar tissue or lines of block. The method may have to be adjusted to handle such anomalies. For example, the delay-based constraint could be imposed only for pairs with a large $\mathrm{CC}$ between aligned signals. In this work, pairs of nodes were formed only within the surface and not transmurally. Nevertheless, the delaybased constraint acting along the surface alone was sufficient to resolve ambiguities between endo- and epicardial sources. While transmural pairs might further improve reconstructions, this also indicates that the constraint offers a very effective regularization that can be used without having to make more uncertain assumptions.

\section{References}

[1] Brooks DH, Ahmad GF, MacLeod RS, Maratos GM. Inverse electrocardiography by simultaneous imposition of multiple constraints. IEEE Trans Biomed Eng 1999;46(1):3-18.

[2] Messnarz B, Tilg B, Modre R, Fischer G, Hanser F. A new spatiotemporal regularization approach for reconstruction of cardiac transmembrane potential patterns. IEEE Trans Biomed Eng 1 2004;51(2):273-281.

[3] Ghodrati A, Brooks DH, Tadmor G, MacLeod RS. Wavefront-based models for inverse electrocardiography. IEEE Trans Biomed Eng 2006;53(9):1821-1831.

[4] Ni Q, MacLeod RS, Lux RL, Taccardi B. A novel interpolation method for electric potential fields in the heart during excitation. Ann Biomed Eng 1998;26(4):597-607.

[5] Matlab code used in this work. https://github.com/ CECGImaging/DelayBasedRegularization.

[6] Geselowitz DB, Miller W. A bidomain model for anisotropic cardiac muscle. Ann Biomed Eng 1983;11(3-4):191-206.

[7] Dubois R, Labarthe S, Coudière Y, Hocini M, Haïssaguerre M. Global and directional activation maps for cardiac mapping in electrophysiology. In Comput Cardiol. IEEE, 2012; 349-352.

[8] Erem B, Brooks DH, van Dam PM, Stinstra JG, MacLeod RS. Spatiotemporal estimation of activation times of fractionated ecgs on complex heart surfaces. In Eng Med Biol Soc. IEEE, 2011; 5884-5887.

[9] Erem B, Coll-Font J, Orellana RM, Št P, Brooks DH, et al. Using transmural regularization and dynamic modeling for noninvasive cardiac potential imaging of endocardial pacing with imprecise thoracic geometry. IEEE Trans Med Imaging 2014;33(3):726-738.

Address for correspondence:

Steffen Schuler, publications@ @it.edu

Fritz-Haber-Weg 1, 76131 Karlsruhe, Germany 